\title{
Prognostic value of long non-coding RNA CRNDE in gastrointestinal cancers: a meta-analysis
}

This article was published in the following Dove Press journal:

Cancer Management and Research

Tian-Yu He

$\mathrm{Si}-\mathrm{Han} \mathrm{Li}$

Jin Huang

Ming Gong

Guang Li

Department of Radiation Oncology, The First Hospital of China Medical

University, Shenyang, Liaoning, People's

Republic of China
Correspondence: Guang $\mathrm{Li}$

Department of Radiation Oncology, The

First Hospital of China Medical University,

Shenyang, Liaoning I I000I, People's

Republic of China

Tel + I 8204080699

Email liguang_cmu@I63.com
Purpose: Numerous studies have reported that the long non-coding RNA colorectal neoplasia differentially expressed (CRNDE) plays important roles in the tumorigenesis, progression, and prognosis of various types of cancer. However, thus far, a systematic analysis of CRNDE in cancers of the digestive system has not been conducted. Thus, the aim of this meta-analysis was to explore the relationship between CRNDE expression and survival or the clinicopathological features of gastrointestinal cancer.

Methods: Eligible studies were collected from nine databases (ie, PubMed, Medline, Embase, Cochrane Library, Ovid, Science Citation Index Expanded, China Biology Medicine, Chinese National Knowledge Infrastructure, and Wanfang). The meta-analysis was conducted using the Stata SE.12 Software. The pooled hazard ratio (HR) or odds ratio (OR) with a $95 \%$ confidence interval $(\mathrm{Cl})$ was used to assess the clinical value of CRNDE expression in gastrointestinal cancers.

Results: A total of 1,053 patients from nine articles were selected. The analysis provided evidence suggesting a significant negative correlation between high CRNDE expression and the rate of overall survival $[\mathrm{HR}=1.92,95 \% \mathrm{CI}(1.40-2.64), p<0.001]$ in patients with malignancies of the digestive system. A positive correlation was observed between high CRNDE expression and lymph node metastasis [OR=2.82, 95\% CI $(1.85-4.31), p<0.001]$, distant metastasis $[\mathrm{OR}=2.72,95 \% \mathrm{CI}(1.16-6.35), p=0.021]$, more advanced tumor-node-metastasis stage $[\mathrm{OR}=3.13,95 \% \mathrm{CI}(2.03-4.83), p<0.001]$, and tumor size $>5 \mathrm{~cm}[\mathrm{OR}=2.81,95 \% \mathrm{CI}$ (1.62-4.88), $p<0.001]$. In the non-colorectal cancer subgroup, high CRNDE expression predicted worse histopathological grade $[\mathrm{OR}=2.21,95 \% \mathrm{CI}(1.37-3.57), p=0.001]$ and depth of tumor invasion [OR=2.54, 95\% CI (1.46-4.41), $p=0.001]$.

Conclusion: This meta-analysis revealed that CRNDE may be an unfavorable risk factor of survival and predict advanced clinicopathological features of patients with gastrointestinal cancer. These findings emphasize the usefulness of CRNDE as a predictor of prognosis and pathological biomarker in this type of tumors.

Keywords: colorectal neoplasia differentially expressed, long non-coding RNA, gastrointestinal cancers, prognosis, clinical pathological features

\section{Introduction}

Gastrointestinal malignancies mainly comprise colorectal cancer (CRC), gastric cancer (GC), hepatocellular carcinoma (HCC), esophageal cancer, pancreatic cancer (PC), and gallbladder cancer. ${ }^{1,2}$ In 2018 , it was estimated that 4,930,000 new cases (27\% of the total number of cases) and 3,530,000 new cancer-related deaths $(37 \%$ of the total cancer deaths) were assigned to malignancies of the digestive system. ${ }^{1,2}$ Digestible system cancers seriously threaten psychological and physical health worldwide. Although marked progress and developments have been achieved in 
the screening, diagnosis, treatment, and prognostic evaluation of digestive system cancers, the rate of long-term survival rate of patients remains unsatisfactory. ${ }^{3-7}$ Thus, it is clinically necessary and urgent to identify reliable biomarkers which can contribute to the screening, diagnosis, and evaluation of prognosis in gastrointestinal tumors.

Long non-coding RNA (lncRNA) is a class of noncoding RNA molecules (length $>200$ nucleotides) without protein-coding potential. ${ }^{8}$ Previously, IncRNA is regarded as a "transcriptional noise". More recently, numerous studies revealed that lncRNAs act as regulators of gene expression and are involved in carcinogenesis, cell proliferation and invasion, or metastasis. ${ }^{9,10}$ Currently, LncRNAs have become a research hotspot in the field of cancer. LncRNA colorectal neoplasia differentially expressed (CRNDE), which is located on chromosome 16, exhibits high levels of expression in CRC, ${ }^{11} \mathrm{GC},{ }^{12}$ glioma, ${ }^{13} \mathrm{HCC},{ }^{14}$ etc. This high expression suggests that CRNDE may be involved in cancer cell proliferation, migration, and invasion, metabolism, angiogenesis, suppression of apoptosis, and tumor occurrence/development. Tissue- or serum-derived CRNDE acts as a biomarker with outstanding sensitivity and specificity.

Numerous studies have demonstrated that the elevated expression of CRNDE in gastrointestinal cancer was associated with poor prognosis, more advanced tumor-nodemetastasis (TNM) stage, and lymph node metastasis. ${ }^{11,15-22}$ In addition, several research studies investigating the association between CRNDE expression and clinicopathological characteristics (ie, distant metastasis, histopathological grade, tumor invasion depth, and tumor size) yielded contradictory results. Jiang et al and Liu et al suggested that high CRNDE expression was associated with a higher risk of distant metastasis; however, Han et al and Xia et al did not report a significant association. ${ }^{11,15,17,22}$ These studies were mostly single-center clinical studies with small sample sizes, investigating the mechanism of a single pathway mechanism. Consequently, the results obtained from individual studies were inconclusive. The objective of this quantitative metaanalysis was to summarize the results of published research studies, aiming to elucidate the relationship between CRNDE expression and survival prognosis of survival or clinicopathological features in patients with digestive system gastrointestinal cancer.

\section{Materials and methods}

\section{Search strategy}

Eligible articles for this meta-analysis (until 3 October 2018) were retrieved from nine databases (ie, PubMed, Medline,
Embase, Cochrane Library, Ovid, China Biology Medicine, Science Citation Index Expanded, China National Knowledge Infrastructure, and WanFang). The terms used in this search were "CRNDE" or "IncRNA CRNDE" or "colorectal neoplasia differentially expressed" and "neoplasm" or "carcinoma" or "tumor" or "cancer". Moreover, the reference lists of relevant articles were manually investigated during retrieval, to avoid missing any potentially eligible studies. The full-text articles published in English and Chinese were included in this meta-analysis.

\section{Inclusion and exclusion criteria}

Inclusion criteria were as follows: [1] patients diagnosed with histologically confirmed gastrointestinal cancer; [2] studies evaluating the relationship between the expression level of CRNDE in tissue or serum specimens and overall survival (OS) or clinicopathological parameters of any type of gastrointestinal cancer; [3] ability to directly or indirectly extract the hazard ratio (HR) with 95\% confidence interval (CI) from survival curves. Exclusion criteria were as follows: [1] unavailability of data regarding survival or clinicopathological features; [2] duplicate or similar studies; [3] reviews, meta-analyses, case reports, and letters not published in English or Chinese; [4] cell or animal laboratory studies.

\section{Data extraction}

The following information was collected from each of the eligible articles: name of first author, year of publication, country, type of cancer, sample source, test method, case number, follow-up time, survival end-point, HR with $95 \%$ $\mathrm{CI}$, and clinicopathological parameters such as age, sex, histopathological grade, tumor invasion depth ( $\mathrm{T}$ stage), lymph node metastasis (N stage), distant metastasis ( $\mathrm{M}$ stage), TNM stage, and tumor size. For studies in which the results of both univariate and multivariate analyses were provided, only the latter data were selected because of the higher precision regarding the interpretation of confounding factors. For research studies providing only Kaplan-Meier curve data, the HRs with $95 \%$ CIs were calculated using the Engauge Digitizer version 10.10. ${ }^{23,24}$ Two investigators independently extracted all the essential data from the selected literature. Discrepancies or disagreements were discussed and overcome in consultation with a third investigator.

\section{Quality assessment}

The quality of all included studies was assessed using the Newcastle-Ottawa Scale. Those with a Newcastle-Ottawa Scale score $\geq 6$ were regarded as high-quality studies. 


\section{Statistical methods}

This meta-analysis was performed using the Stata SE12.0 software (StataCorp, College Station, Texas). A $P<0.05$ denoted statistical significance. HRs with 95\% CIs were calculated to assess the relationship between CRNDE expression and survival risk. Odds ratios (ORs) with 95\% CIs were calculated to assess the relationship between CRNDE expression and clinicopathological features (ie, age, sex, histopathological grade, $\mathrm{T}$ stage, $\mathrm{N}$ stage, $\mathrm{M}$ stage, TNM stage, and tumor size). The heterogeneity among studies was evaluated using the chi-squared-based $\mathrm{Q}$ test and $\mathrm{I}^{2}$ statistics. In cases of extreme heterogeneity (ie, $\mathrm{I}^{2}>50 \%$ or $P<0.10$ for $\mathrm{Q}$ test), we used the random-effects model. Otherwise, the fixed effects model was applied. The subgroup analysis was performed based on different items. The sensitivity analysis was performed to evaluate the accuracy and robustness of the results. Publication bias analysis was performed using the Begg's funnel plot and Egger's test. A two-sided $P<0.1$ denoted statistical significance, for which the Duval and Tweedie's trim and fill method was applied.

\section{Results}

\section{Characteristics of eligible studies}

According to the inclusion and exclusion criteria, a total of nine eligible studies were finally included in the current meta- analysis. These included eight and seven studies of prognosis and clinicopathological characteristics. A total of 1,053 patients were included in these studies, with a minimum and maximum sample size of 58 and 251 patients, respectively. Notably, eight and one studies were published in English and Chinese, respectively. Four tumor types were evaluated, including CRC (6), ${ }^{11,15-19} \mathrm{GC}(1),{ }^{20} \mathrm{PC}(1),{ }^{21}$ and intrahepatic cholangiocarcinoma (1). ${ }^{22}$ All studies were performed in China, and all participants were Asian. The detailed process of literature search and selection is presented in Figure 1. The relevant information of the research studies included for the analysis of prognosis and clinicopathological parameters is shown in Tables 1 and 2, respectively.

\section{Association between CRNDE and prognosis}

A total of 973 patients from nine studies were included in this meta-analysis. ${ }^{11,15-17,19-22}$ The heterogeneity test revealed high heterogeneity $\left(\mathrm{I}^{2}=61.5 \%, p=0.011\right)$. The random effects model was used to analyze the pooled HRs with $95 \%$ CI. The results revealed a significant association between high CRNDE expression and poor OS $[\mathrm{HR}=1.92,95 \%$ CI (1.40-2.64), $p<0.001]$ in gastrointestinal cancer (Figure 2A).

A meta-analysis of the subgroups was further performed based on the type of cancer, sample source, sample size,

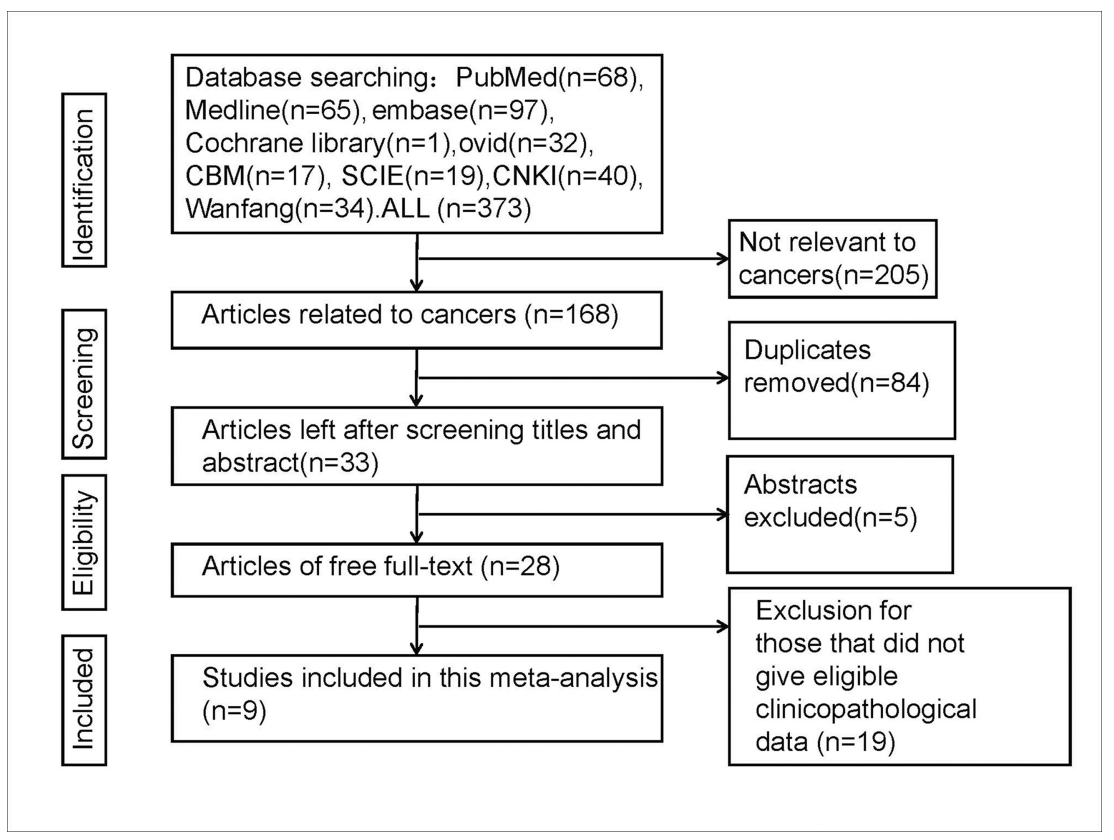

Figure I Flowchart of the current meta-analysis. 


\begin{tabular}{|c|c|c|c|}
\hline 㺼 & $\infty$ & $\infty$ & $\begin{array}{lllllll}\infty & \infty & \infty & \infty & 0 & \infty & \infty\end{array}$ \\
\hline  & $\stackrel{y}{\check{\nu}}$ & 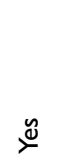 & 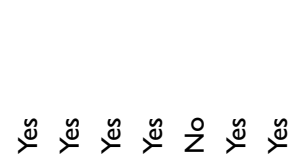 \\
\hline 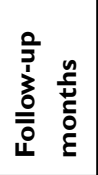 & 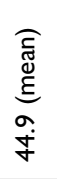 & 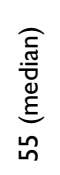 & 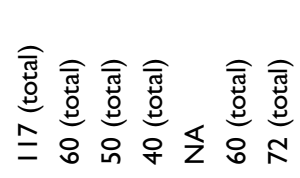 \\
\hline 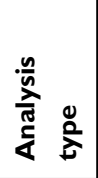 &  & 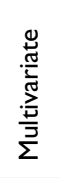 & 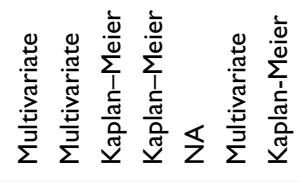 \\
\hline 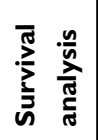 & Oั & oิ & 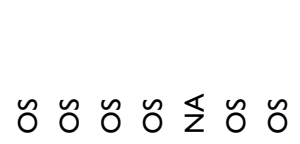 \\
\hline 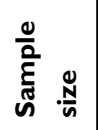 & $\stackrel{\infty}{+}$ & $\underline{q}$ & 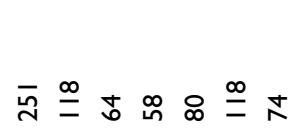 \\
\hline ن̃ & 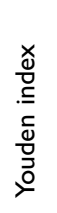 & 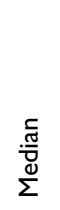 & 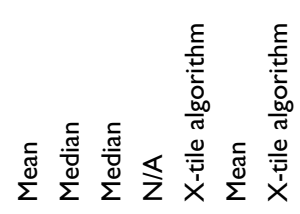 \\
\hline 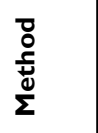 & 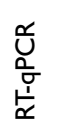 & 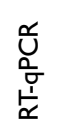 & 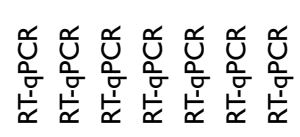 \\
\hline 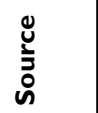 & 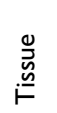 & 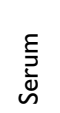 & 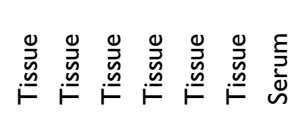 \\
\hline 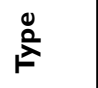 & Ữ & 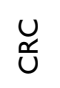 & 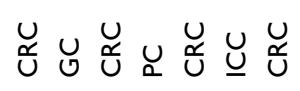 \\
\hline 仓ે & : & $\stackrel{\widetilde{n}}{\overline{\bar{U}}}$ & 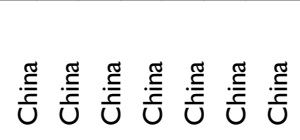 \\
\hline ঠ & $\frac{0}{2}$ & $\frac{0}{2}$ & 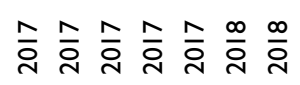 \\
\hline 高 & \multicolumn{3}{|c|}{ 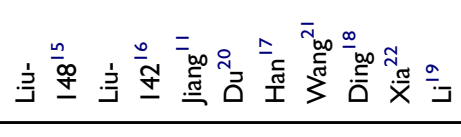 } \\
\hline
\end{tabular}

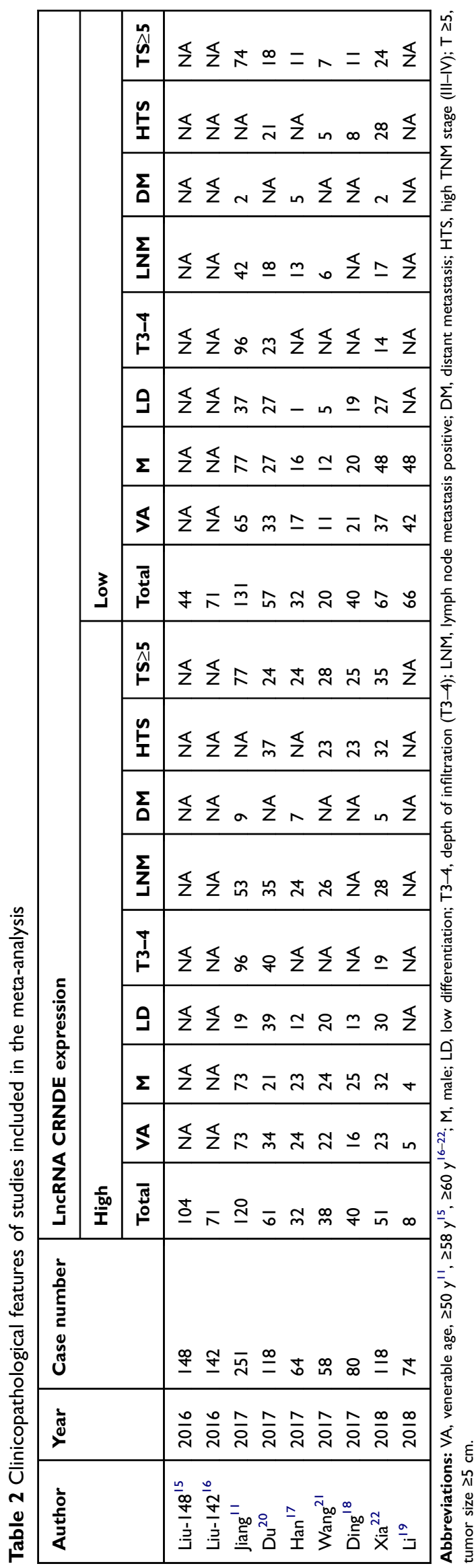


A

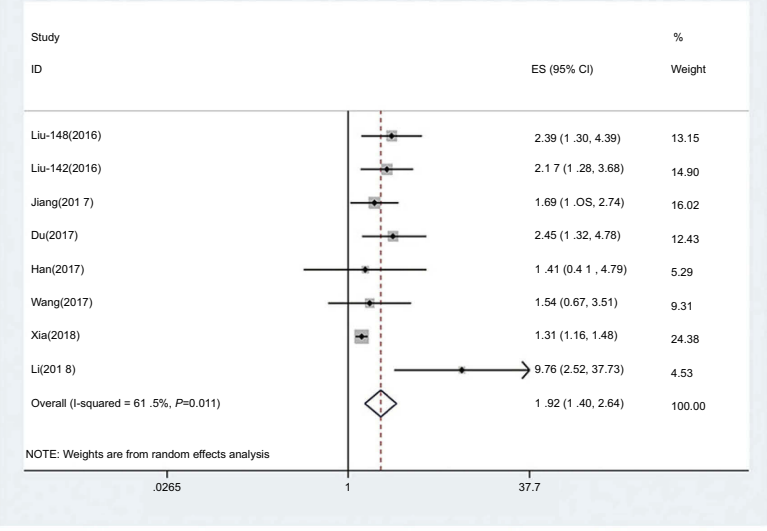

C

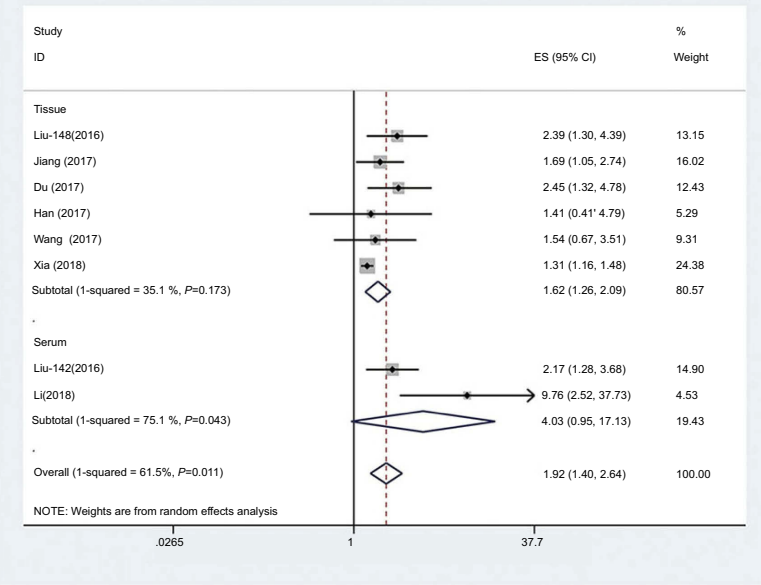

B

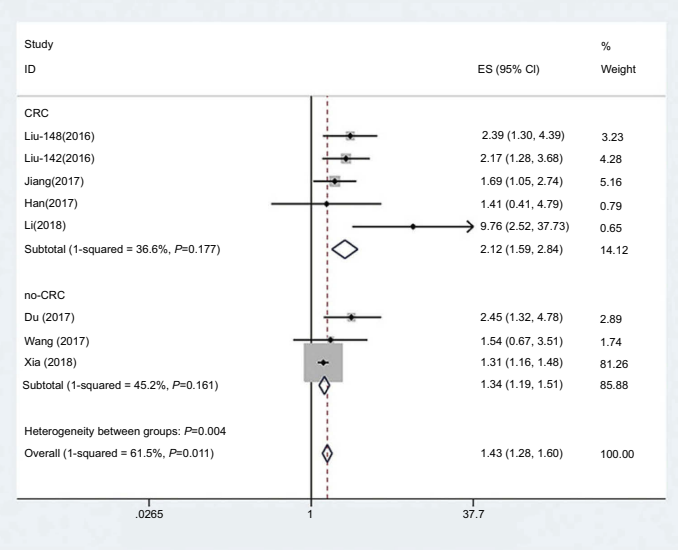

Figure 2 Meta-analysis of the pooled HRs of OS in different types of cancer with high CRNDE expression. (A) Forest plot of the correlation between CRNDE expression and OS. (B) Subgroup analysis of HRs of OS according to the factor of type of cancer. (C) Subgroup analysis of HRs of OS according to the factor of sample source. Abbreviations: CRC, colorectal cancer; no-CRC, non-colorectal cancer; ES, effect size.

follow-up times, and type of analysis (Table 3). A negative correlation was observed between high CRNDE expression and $\mathrm{OS}$, in patients with both $\mathrm{CRC}[\mathrm{HR}=2.12,95 \% \mathrm{CI}$ (1.59-2.84), $p<0.001]$ and non-CRC $[\mathrm{HR}=1.34,95 \% \mathrm{CI}$ (1.19-1.51), $p=0.024]$ (Figure 2B). In addition, a negative correlation was observed between high CRNDE expression and $\mathrm{OS}$ in samples derived from tissue $[\mathrm{HR}=1.62,95 \% \mathrm{CI}$ (1.26-2.09), $p<0.001]$ (Figure 2C). Moreover, a negative correlation was observed between high CRNDE expression and OS in the sample size $\geq 100$ subgroup $[\mathrm{HR}=1.80,95 \% \mathrm{CI}$ (1.32-2.46), $p<0.001]$ (Figure S1A). A negative correlation was also observed between high CRNDE expression and OS in patients with follow-up times $<5$ years $[\mathrm{HR}=2.04,95 \% \mathrm{CI}$ (1.45-2.88), $p<0.001]$ and $\geq 5$ years $[\mathrm{HR}=2.02,95 \% \mathrm{CI}$ (1.21-3.39), $p=0.008$ ] (Figure S1B). In studies in which a multivariate analysis was reported, the association between high CRNDE expression and OS of patients was significant $[\mathrm{HR}=1.80,95 \% \mathrm{CI}(1.32-2.46), p<0.001]$ (Figure S1C). There was no significant association observed in other subgroups.
The sensitivity analysis suggested that the pooled HR was not significantly affected by any individual study. Therefore, the conclusions derived from the present analysis were accurate and robust (Figure 3).

\section{Association between CRNDE and clinicopathological features}

Seven studies, including a total of 763 patients, provided data regarding clinicopathological parameters. ${ }^{11,17-22}$ The fixed effects or random models were used to analyze the relationship between the CRNDE expression levels and clinicopathological parameters (Table 4). The pooled effect estimates of the $\mathrm{N}$ stage $[\mathrm{OR}=2.82, \quad 95 \% \quad \mathrm{CI} \quad(1.85-4.31), \quad p<0.001]$ (Figure 4A), $M$ stage $[\mathrm{OR}=2.72,95 \%$ CI $(1.16-6.35)$, $p=0.021]$ (Figure 4B), more advanced TNM stage $[\mathrm{OR}=3.13$, 95\% CI (2.03-4.83), $p<0.001]$ (Figure 4C), tumor size $\geq 5 \mathrm{~cm}$ $[\mathrm{OR}=2.81,95 \% \mathrm{CI}(1.62-4.88), p<0.001]$ (Figure 4D) were in favor of the high CRNDE expression group. 
Table 3 Subgroup analysis of overall survival

\begin{tabular}{|c|c|c|c|c|c|c|c|c|}
\hline \multirow[t]{2}{*}{ Subgroups } & \multirow[t]{2}{*}{ Studies(n) } & \multirow[t]{2}{*}{ Number of patients } & \multirow[t]{2}{*}{ HR } & \multirow[t]{2}{*}{$95 \% \mathrm{Cl}$} & \multirow[t]{2}{*}{$P$} & \multicolumn{3}{|c|}{ Heterogeneity } \\
\hline & & & & & & $I^{2}(\%)$ & $P$ & Model \\
\hline Total & 8 & 793 & 1.92 & $1.40-2.64$ & $<0.001$ & $61.5 \%$ & 0.011 & Random \\
\hline \multicolumn{9}{|l|}{ Cancer type } \\
\hline CRC & 5 & 679 & 2.12 & $1.59-2.84$ & $<0.001$ & $36.6 \%$ & 0.177 & Fixed \\
\hline Non-CRC & 3 & 294 & 1.34 & $|| 9-.|.5|$ & 0.024 & $45.2 \%$ & 0.161 & Fixed \\
\hline \multicolumn{9}{|l|}{ Source } \\
\hline Tissue & 6 & 757 & 1.62 & $1.26-2.09$ & $<0.001$ & $35.1 \%$ & 0.173 & Random \\
\hline Serum & 2 & 216 & 4.03 & $0.95-17.13$ & 0.059 & $75.7 \%$ & 0.043 & Random \\
\hline \multicolumn{9}{|l|}{ Sample size } \\
\hline$\geq 100$ & 5 & 777 & 1.80 & $1.32-2.46$ & $<0.001$ & $61.3 \%$ & 0.035 & Random \\
\hline$<100$ & 3 & 196 & 2.56 & $0.84-7.78$ & 0.098 & $65.9 \%$ & 0.053 & Random \\
\hline \multicolumn{9}{|l|}{ Follow-up } \\
\hline$<5$ & 4 & 412 & 2.04 & $1.45-2.88$ & $<0.001$ & $0.0 \%$ & 0.776 & Random \\
\hline$\geq 5$ & 4 & 561 & 2.02 & $1.21-3.39$ & 0.008 & $76.1 \%$ & 0.006 & Random \\
\hline \multicolumn{9}{|l|}{ Analysis type } \\
\hline Multivariate analysis & 5 & 777 & 1.80 & $1.32-2.46$ & $<0.001$ & $61.3 \%$ & 0.035 & Random \\
\hline Kaplan-Meier & 3 & 196 & 2.56 & $0.84-7.78$ & 0.098 & $65.9 \%$ & 0.053 & Random \\
\hline
\end{tabular}

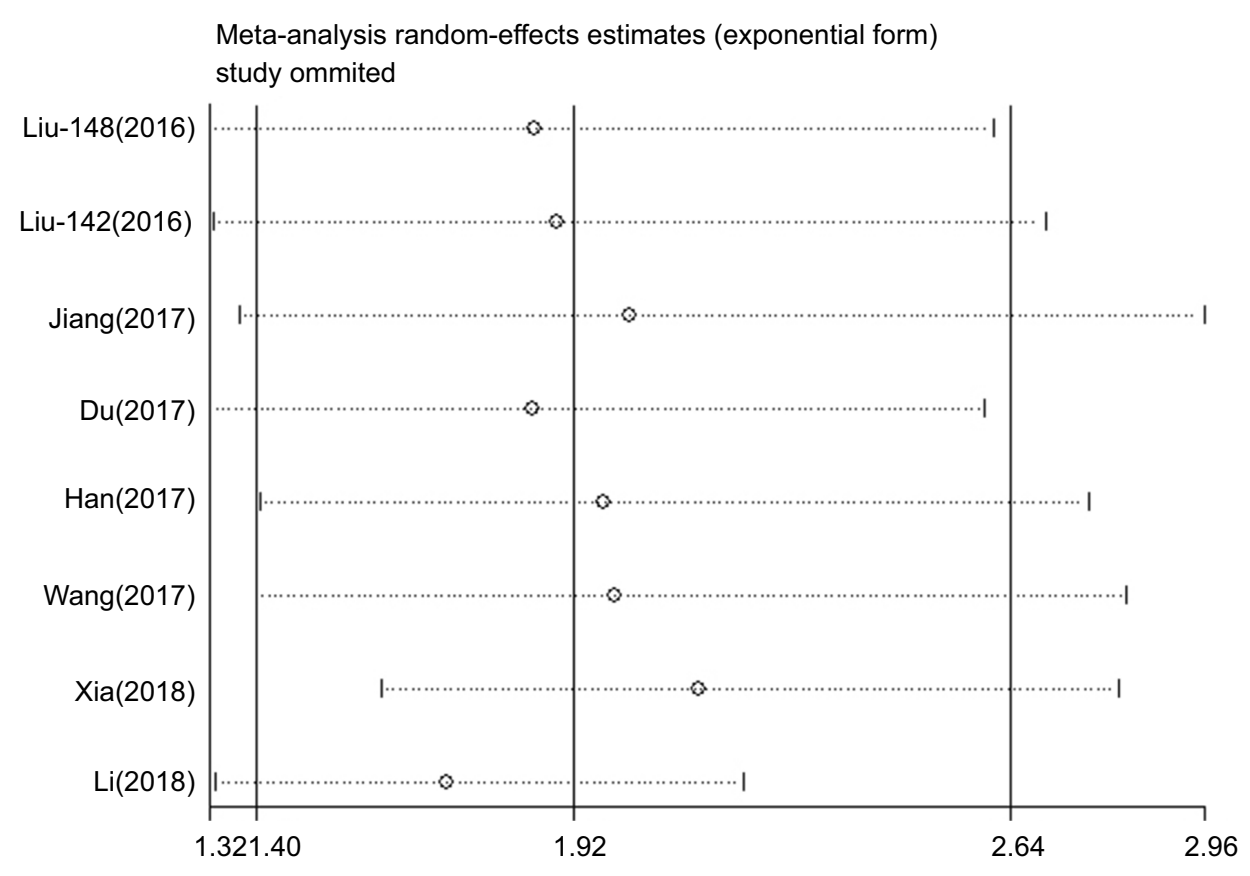

Figure 3 Sensitivity of CRNDE expression for overall survival.

In the non-CRC group, a significant association between high CRNDE expression and high $\mathrm{T}$ stage $[\mathrm{OR}=2.54,95 \% \mathrm{CI}$ (1.46-4.41), $p=0.001]$ (Figure 4E) or worse histopathological grade [OR $=2.21,95 \%$ CI $(1.37-3.57), p=0.001]$ (Figure 4F) was observed. Nevertheless, there was no association observed with age (Figure S2A) or sex (Figure S2B). 
Table 4 Subgroup analysis of pooled odds ratios for the relationship between CRNDE expression levels and clinicopathological parameters by factor of cancer type

\begin{tabular}{|c|c|c|c|c|c|c|c|c|}
\hline \multirow[t]{2}{*}{ Category } & \multirow{2}{*}{$\begin{array}{l}\text { Studies } \\
\text { (n) }\end{array}$} & \multirow{2}{*}{$\begin{array}{l}\text { Number of } \\
\text { patients }\end{array}$} & \multirow[t]{2}{*}{ OR } & \multirow[t]{2}{*}{$95 \% \mathrm{Cl}$} & \multirow[t]{2}{*}{$P$} & \multicolumn{3}{|c|}{ Heterogeneity } \\
\hline & & & & & & $I^{2}(\%)$ & $P$ & Model \\
\hline Age (venerable age vs no) & 7 & 763 & I.II & $0.83-1.50$ & 0.486 & $29.1 \%$ & 0.206 & Fixed \\
\hline CRC & 4 & 469 & 1.36 & $0.92-2.01$ & 0.124 & $43.5 \%$ & 0.151 & Fixed \\
\hline Non-CRC & 3 & 294 & 0.84 & $0.52-1.33$ & 0.451 & $0.0 \%$ & 0.699 & Fixed \\
\hline Sex (female vs male) & 7 & 763 & 1.00 & $0.74-1.36$ & 0.997 & $29.5 \%$ & 0.203 & Fixed \\
\hline CRC & 4 & 469 & 0.79 & $0.54-1.77$ & 0.240 & $24.9 \%$ & 0.262 & Fixed \\
\hline Non-CRC & 3 & 294 & 1.43 & $0.88-2.32$ & 0.144 & $0.0 \%$ & 0.608 & Fixed \\
\hline Differentiation (poor vs well-morderate) & 5 & 609 & 2.03 & $0.79-5.20$ & 0.140 & $81.2 \%$ & 0.000 & Random \\
\hline CRC & 2 & 315 & 2.56 & $0.07-92.17$ & 0.140 & $90.6 \%$ & 0.001 & Random \\
\hline Non-CRC & 3 & 294 & 2.21 & $1.37-3.57$ & 0.001 & $0.0 \%$ & 0.756 & Random \\
\hline Depth of infiltration (T3-4 vs TI-2) & 4 & 567 & 1.53 & $0.8 I-2.9 \mid$ & 0.191 & $65.2 \%$ & 0.035 & Random \\
\hline CRC & 2 & 331 & 0.94 & $0.35-2.50$ & 0.895 & $70.0 \%$ & 0.068 & Random \\
\hline Non-CRC & 2 & 236 & 2.54 & $|.46-4.4|$ & 0.001 & $0.0 \%$ & 0.690 & Random \\
\hline Lymph node metastasis (positive vs negative) & 5 & 609 & 2.82 & $|.85-4.3|$ & $p<0.001$ & $29.7 \%$ & 0.224 & Random \\
\hline CRC & 2 & 315 & 2.41 & $0.97-6.01$ & 0.059 & $60.5 \%$ & 0.111 & Random \\
\hline Non-CRC & 3 & 294 & 3.49 & $2.13-5.70$ & $p<0.001$ & $0.0 \%$ & 0.740 & Random \\
\hline Distant metastasis (yes vs no) & 3 & 433 & 2.72 & $1.16-6.35$ & 0.021 & $0.0 \%$ & 0.450 & Fixed \\
\hline CRC & 2 & 315 & 2.49 & $0.93-6.64$ & 0.069 & $32.0 \%$ & 0.225 & Fixed \\
\hline Non-CRC & 1 & 118 & 3.53 & $0.66-19.0$ & 0.141 & - & - & Fixed \\
\hline TNM stage (III-IV vs I-II) & 4 & 374 & 3.13 & $2.03-4.83$ & $p<0.001$ & $0.0 \%$ & 0.508 & Fixed \\
\hline CRC & I & 80 & 5.41 & $2.00-14.66$ & 0.001 & - & - & Fixed \\
\hline Non-CRC & 3 & 294 & 2.75 & $1.70-4.46$ & $p<0.001$ & $0.0 \%$ & 0.642 & Fixed \\
\hline Tumor size $(\geq 5 \mathrm{vs}<5 \mathrm{~cm})$ & 6 & 689 & 2.81 & $1.62-4.88$ & $p<0.001$ & $62.5 \%$ & 0.020 & Random \\
\hline CRC & 3 & 395 & 2.99 & $1.13-7.93$ & $p<0.001$ & $75.8 \%$ & 0.016 & Random \\
\hline Non-CRC & 3 & 294 & 2.79 & $1.29-6.05$ & $p<0.001$ & $57.0 \%$ & 0.098 & Random \\
\hline
\end{tabular}

The sensitivity analysis revealed that the pooled ORs were not significantly affected by any individual study. Therefore, the conclusions derived from this analysis in both the whole group and subgroups were accurate and robust (Figure $\mathrm{S} 3 \mathrm{~A}-\mathrm{H}$ ).

\section{Publication bias}

Begg's funnel plot and Egger's regression test suggested that there was no publication bias across the clinicopathological features, except for $\mathrm{N}$ stage (Begg's test: $p=0.027$, Egger's test: $p=0.011$ ). For the subgroups (Begg's test: $p<0.05$, Egger's test: $p<0.05$ ), the Duval and Tweedie's trim and fill method was applied. The results showed that the adjusted values were similar to the observed ones (Table 5).

\section{Discussion}

The incidence and mortality associated with gastrointestinal tumors is constantly increasing. Gastrointestinal cancers account for $27 \%$ and $37 \%$ of the total number of new cases and cancer-related death, respectively. Among them, CRC contributes $10 \%$ and $9 \%$ of the total number of new cases and cancer deaths, respectively, ranking third in terms of incidence and second in terms of mortality. ${ }^{1}$ Therefore, it is particularly important to identify highly sensitive and specific biomarkers for the accurate diagnosis, treatment, and evaluation of prognosis in patients with gastrointestinal tumors.

Via interaction with DNA, RNA, proteins, lipids, or other molecules, IncRNAs act as key signal transduction mediators in cancer signaling pathways, and exert an effect 
A

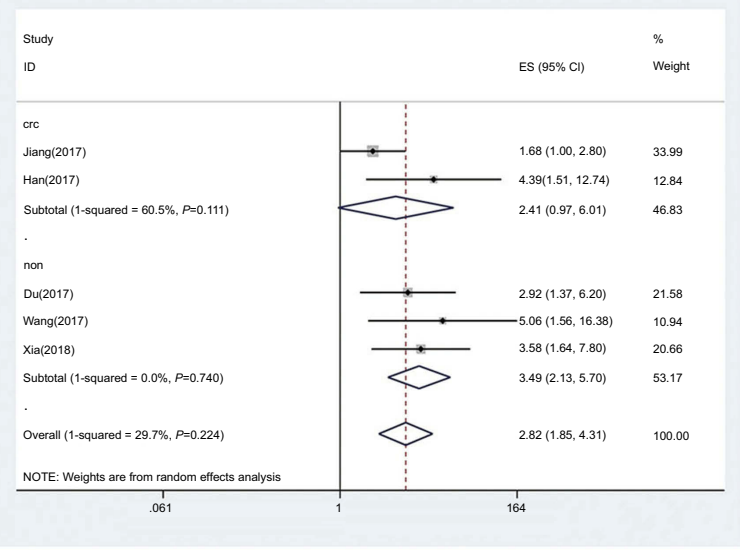

C

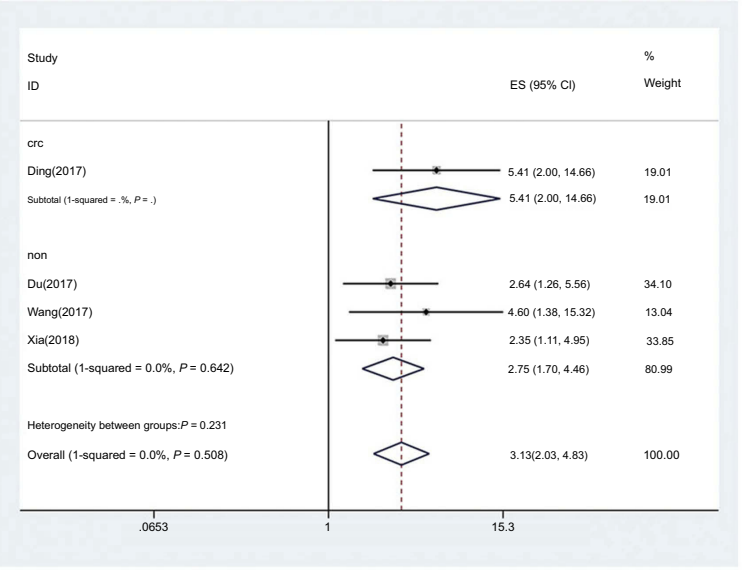

E

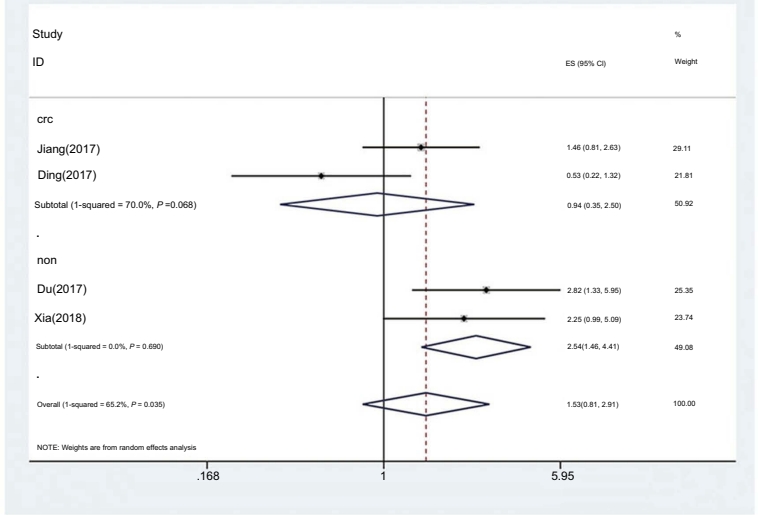

B

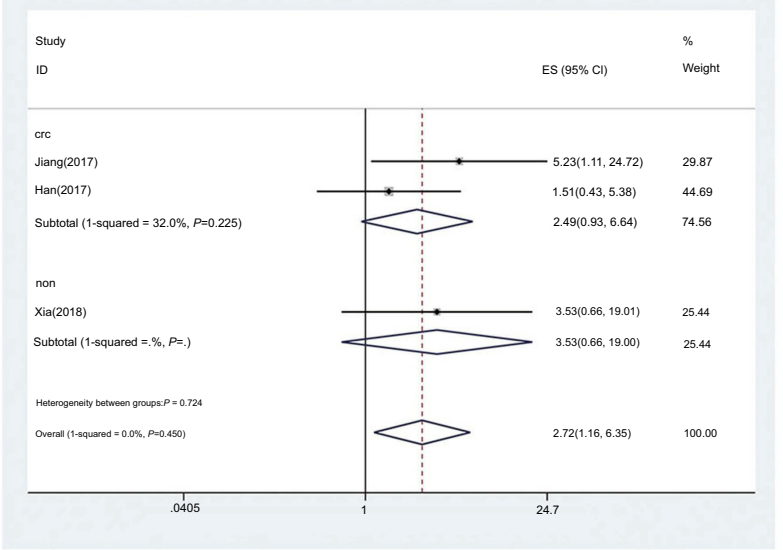

D

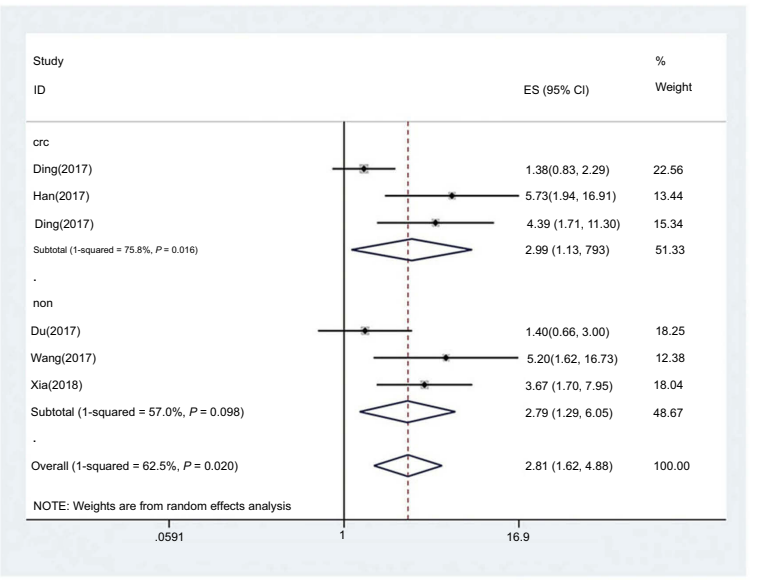

$\mathbf{F}$



Figure 4 Forest plot of the correlation between CRNDE expression and clinicopathological characteristics. (A) Lymph node metastasis (positive vs negative). (B) Distant metastasis (yes vs no). (C) TNM stage (III-IV vs I-II). (D) Tumor size ( $\geq 5$ vs $<5 \mathrm{~cm}$ ). (E) Depth of infiltration (T3-4 vs TI-2). (F) Differentiation (poor vs well-moderate). Abbreviations: CRC, colorectal cancer; non, non-colorectal cancer; ES, effect size.

on proliferation, metabolism, invasion, radioresistance, and chemoresistance. ${ }^{25-27}$ Recent discoveries have revealed lncRNAs contributed to the diagnosis, treatments, and prognosis of cancer patients. For example, Liu et al suggested that the combination of CRNDE and carcino-embryonic antigen (CEA) expression showed improved diagnostic value versus each expression alone. ${ }^{15}$ Zhang et al suggested that CRNDE contributed to the formation of the radioresistant phenotype of lung adenocarcinoma cells as an oncogene by modulating $\mathrm{p} 21 .{ }^{26} \mathrm{Qu}$ et al suggested that activated in RCC with sunitinib resistance (ARSR) enhanced resistance to sunitinib by competitively binding miR-34/miR-449 in renal cell cancer. ${ }^{27}$ 
Table 5 The results of Begg's and Egger's tests for the publication bias

\begin{tabular}{|c|c|c|c|c|c|c|c|}
\hline & \multicolumn{2}{|c|}{ Begg's test } & \multicolumn{3}{|c|}{ Duval and Tweedie's trim and fill } & \multicolumn{2}{|c|}{ Egger's test } \\
\hline & $\mathbf{Z}$ & $\boldsymbol{P}$ & Trimmed studies & Observed values $(\mathrm{Cl})$ & Adjusted values (CI) & Intercept & $P$ \\
\hline HR analysis & 0.870 & 0.386 & 5 & $1.92(1.40-2.64)$ & $1.33(1.00-1.76)$ & 1.734 & 0.013 \\
\hline Age & 0.000 & 1.000 & - & - & - & -0.652 & 0.685 \\
\hline Sex & 0.300 & 0.764 & - & - & - & -0.164 & 0.921 \\
\hline Differentiation & 2.200 & 0.027 & 2 & $2.03(0.79-5.20)$ & $1.24(0.52-2.97)$ & 4.684 & 0.119 \\
\hline Depth of infiltration & 0.340 & 0.734 & - & - & - & -2.573 & 0.716 \\
\hline Lymph node metastasis & 2.200 & 0.027 & 2 & $2.82(I .85-4.3 I)$ & $2.42(1.63-3.58)$ & 3.507 & 0.011 \\
\hline Distant metastasis & 0.000 & 1.000 & - & - & - & 5.711 & 0.277 \\
\hline TNM stage & 0.340 & 0.734 & - & - & - & 3.469 & 0.127 \\
\hline Tumor size & 1.880 & 0.060 & 0 & $2.81(1.62-4.88)$ & $2.81(1.62-4.88)$ & 4.537 & 0.024 \\
\hline
\end{tabular}

The results of this meta-analysis confirmed that high CRNDE expression may serve as a negative risk factor of survival, and predict advanced clinicopathological characteristics of patients with gastrointestinal tumors. According to the cancer type subgroup analysis, CRNDE could predict worse prognosis in CRC, but not in non-CRC. Based on the sample source subgroup analysis, CRNDE could predict poor prognosis in the tissue group; however, this ability was inconsistent in the serum group. This result may be attributed to the lack of relevant research on serum samples. Additional clinical trials are warranted to address the lack of data. Furthermore, the relationship between the expression of CRNDE and clinicopathological characteristics was investigated in patients. The pooled results revealed that high CRNDE expression was positively correlated with more advanced TNM stage and tumor size $\geq 5 \mathrm{~cm}$. This correlation was stronger in CRC patients. High CRNDE expression was positively correlated with lymph node metastases. This correlation was stronger in non-CRC patients. Collectively, a positive correlation was observed between high CRNDE expression and $\mathrm{M}$ stage and $\mathrm{N}$ stage. However, this relationship was not observed in the cancer type subgroup analysis. This discrepancy may be attributed to the limited number of studies included in this analysis. Considering the high OR value recorded in the overall analysis, the high expression of CRNDE exhibits a great potential for the prediction of distant metastasis. Of note, in the CRC group, there was no significant correlation between CRNDE expression and worse histopathological grade or T-stage. However, in the non-CRC group, a positive correlation was observed between these factors. There was no significant association observed between CRNDE expression and age and sex.
Thus far, all published studies confirmed that high CRNDE expression was associated with a shorter OS in patients. These findings are consistent with the results observed in the present meta-analysis. The HRs reported by $\mathrm{Li}$ et al were higher than those of other studies. An explanation may be that patients in the study reported by $\mathrm{Li}$ et al exhibited a more advanced TNM stage and received FOLFOX chemotherapy versus patients in other studies (TNM stage I-IV). ${ }^{19}$ Inter-study heterogeneity mainly originated from this difference. It is suggested that CRNDE expression has a higher prognostic value in patients with more advanced TNM stage. Two meta-analyses investigated the relationship between CRNDE and survival or clinicopathological features in patients with solid cancer. Consistent with the present findings, Xie et al and Liang et al confirmed that high CRNDE expression predicts poor OS. ${ }^{28,29}$ Moreover, both studies suggested that high CRNDE expression is related to more advanced TNM stage and $\mathrm{N}$ stage in patients with solid tumors - a finding which is also consistent with the conclusions of our meta-analysis. Liang et al also suggested that there was no correlation between CRNDE expression and tumor size or histopathological grade. ${ }^{29}$ This observation was different from our results. In their studies, other clinicopathological features (eg, M stage and T stage) were not evaluated. We included more studies, detailed subgroups [cancer type (CRC vs other gastrointestinal cancers), follow-up ( $<5$ years vs $\geq 5$ years), source (tissue vs serum), etc.], and clinicopathological features (age, differentiation, distant metastasis, etc.). In addition, we conducted a more comprehensive and detailed investigation of the relevance of CRNDE to prognosis and clinicopathological characteristics. 
In gastrointestinal tumors, high CRNDE expression is often related to poor OS and more advanced clinicopathological features. Recent research provided novel insights into the functions of CRNDE in cancer pathogenesis and clinicopathological processes. In CRC, CRNDE enhanced cell proliferation and chemoresistance via modulation of Wnt/ $\beta$-catenin through competitively binding miR-217 and miR-181a-5p. ${ }^{17,30}$ CRNDE functioned as a competing endogenous RNA for miR-136, led to the derepression of its endogenous target, E2F transcription factor 1 (E2F1). ${ }^{31}$ Several studies demonstrated that CRNDE regulates tumor progression through interaction with proteins. Jiang demonstrated that CRNDE stabilized by heterogeneous nuclear ribonucleoprotein U-like 2 protein (hnRNPUL2) enhanced cell proliferation and migration by activating the Ras/MAPK signaling pathways. ${ }^{11}$ The results of the present study suggested that CRNDE is involved in cell carcinogenesis and inhibition of apoptosis by binding enhancer of zeste homolog 2 (EZH2). ${ }^{18}$ In GC, the present research confirmed that CRNDE enhanced cellular proliferation by increasing the expression of downstream molecules of E2F transcription factor 3 (E2F3) through derepression of miR-145. ${ }^{12} \mathrm{Du}$ et al indicated that CRNDE accelerated cell migration and invasion via the activation of the PI3K/Akt signaling pathways. ${ }^{20}$ In PC, CRNDE sponges miR-384 to enhance cellular proliferation and metastasis via upregulation of insulin receptor substrate 1 (IRS1). ${ }^{21}$ In HCC, CRNDE suppressed miR384 to promote cellular migration, invasion, and proliferation via the upregulation of NF- $\mathrm{KB}$ and $\mathrm{p}-\mathrm{AKT} .^{32}$ Wang found that CRNDE increased the expression levels of mitogen-activated protein kinase 1 (MAPK1) through inhibition of miR-217 in the process of cell proliferation, migration, and invasion. ${ }^{33} \mathrm{Xia}$ et al demonstrated that CRNDE played a key role in epithelial-mesenchymal transition and metastasis. ${ }^{22}$

The present meta-analysis was characterized by limitations. Firstly, all studies were conducted in China, which may limit the representativeness of other ethnic populations. Thus, international studies with larger sample sizes are necessary to verify the results obtained in this meta-analysis. Secondly, our analysis included only studies published in English or Chinese. This may cause publication bias. Thirdly, partial HRs were calculated or extracted from reconstructed survival curves rather than being directly extracted from the primary studies. Fourthly, the inclusion of research studies without a consistent CRNDE cut-off value was one of the sources of heterogeneity. Finally, owing to the lack of relevant data, it is not possible to analyze the relationship between CRNDE expression and several clinicopathological features, such as CEA expression and tumor location.

\section{Conclusion}

This meta-analysis revealed that CRNDE might act as an unfavorable risk factor of survival and contribute to the elucidation of the relationship between CRNDE and clinicopathological features in gastrointestinal cancer. Collectively, CRNDE may act as a putative cancer biomarker and can be potentially applied for early diagnosis, targeted therapy, and evaluation of prognosis in patients with gastrointestinal cancer. Further studies are warranted to confirm these findings. Future research may determine the appropriate CRNDE cut-off value, develop an efficient and accurate method for serum testing, and elucidate the molecular mechanisms of CRNDE involvement in the promotion of tumorigenesis.

\section{Abbreviation list}

lncRNA, long noncoding RNA; CRNDE, colorectal neoplasia differentially expressed; HR, hazard ratio; OR, odds ratios; OS, overall survival; $95 \% \mathrm{CI}, 95 \%$ confidence intervals; NOS, the Newcastle-Ottawa Quality Assessment Scale; qRT-PCR, quantitative real-time PCR; CRC, colorectal cancer; GC, gastric cancer; HCC, hepatocellular carcinoma; EC, esophageal cancer; PC, pancreatic cancer; GCa, gallbladder cancer; ICC, intrahepatic cholangiocarcinoma; CEA, carcino-embryonic antigen; ARSR, activated in RCC with Sunitinib Resistance; E2F1, E2F Transcription Factor 1; E2F3, E2F transcription factor 3; hnRNPUL2, heterogeneous nuclear ribonucleoprotein U-like 2 protein; EZH2, enhancer of zeste homolog 2; IRS1, insulin receptor

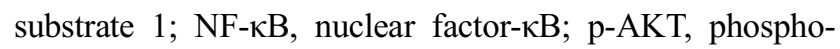
AKT; MAPK1, mitogen-activated protein kinase 1; EMT, epithelial-mesenchymal transition.

\section{Ethics approval and informed consent}

Patients or clinical samples were not investigated in this study; hence, ethical approval was not required.

\section{Acknowledgments}

We would like to thank the researchers and study participants for their contributions. The authors did not receive funding for this research. 


\section{Author contributions}

All authors contributed to data analysis, drafting or revising the article, gave final approval of the version to be published, and agree to be accountable for all aspects of the work.

\section{Disclosure}

The authors report no conflicts of interest in this work.

\section{References}

1. Bray F, Ferlay J, Soerjomataram I, et al. Global cancer statistics 2018 : GLOBOCAN estimates of incidence and mortality worldwide for 36 cancers in 185 countries. CA Cancer J Clin. 2018;68(6):394-424. doi: 10.3322/caac. 21492

2. Siegel RL, Miller KD, Jemal A. Cancer statistics, 2018. CA Cancer J Clin. 2018;68(1):7-30. doi:10.3322/caac.21442

3. Smith RA, Andrews KS, Brooks D, et al. Cancer screening in the United States, 2017: a review of current American Cancer Society guidelines and current issues in cancer screening. CA Cancer J Clin. 2017;67(2):100-121. doi:10.3322/caac.21392

4. Wyld L, Audisio RA, Poston GJ. The evolution of cancer surgery and future perspectives. Nat Rev Clin Oncol. 2015;12(2):115-124. doi:10.1038/nrclinonc.2014.191

5. Kang YK, Boku N, Satoh T, et al. Nivolumab in patients with advanced gastric or gastro-oesophageal junction cancer refractory to, or intolerant of, at least two previous chemotherapy regimens (ONO-4538-12, ATTRACTION-2): a randomised, double-blind, placebo-controlled, phase 3 trial. Lancet. 2017;390(10111):2461-2471. doi:10.1016/ S0140-6736(17)31827-5

6. Valle JW, Lamarca A, Goyal L, Barriuso J, Zhu AX. New horizons for precision medicine in biliary tract cancers. Cancer Discov. 2017;7 (9):943-962. doi:10.1158/2159-8290.CD-17-0245

7. Adiseshaiah PP, Crist RM, Hook SS, McNeil SE. Nanomedicine strategies to overcome the pathophysiological barriers of pancreatic cancer. Nat Rev Clin Oncol. 2016;13(12):750-765. doi:10.1038/nrclinonc.2016.119

8. Ulitsky I. Evolution to the rescue: using comparative genomics to understand long non-coding RNAs. Nat Rev Genet. 2016;17 (10):601-614. doi:10.1038/nrg.2016.85

9. Prensner JR, Chinnaiyan AM. The emergence of lncRNAs in cancer biology. Cancer Discov. 2011;1(5):391-407. doi:10.1158/2159-8290. CD-11-0209

10. Schmitt AM, Chang HY. Long noncoding RNAs in cancer pathways. Cancer Cell. 2016;29(4):452-463. doi:10.1016/j.ccell.2016.03.010

11. Jiang H, Wang Y, Ai M, et al. Long noncoding RNA CRNDE stabilized by hnRNPUL2 accelerates cell proliferation and migration in colorectal carcinoma via activating Ras/MAPK signaling pathways. Cell Death Dis. 2017;8(6):e2862. doi:10.1038/cddis.2017.518

12. Hu CE, Du PZ, Zhang HD, Huang GJ. Long noncoding RNA CRNDE promotes proliferation of gastric cancer cells by targeting miR-145. Cell Physiol Biochem. 2017;42(1):13-21. doi:10.1159/000477107

13. Zheng J, Liu X, Wang P, et al. CRNDE promotes malignant progression of glioma by attenuating miR-384/PIWIL4/STAT3 axis. Mol Ther. 2016;24(7):1199-1215. doi:10.1038/mt.2016.71

14. Ji D, Jiang C, Zhang L, et al. LncRNA CRNDE promotes hepatocellular carcinoma cell proliferation, invasion, and migration through regulating miR-203/BCAT1 axis. J Cell Physiol. 2018;234(5):65486560. doi:10.1002/jcp. 27396

15. Liu T, Zhang X, Yang YM, et al. Increased expression of the long noncoding RNA CRNDE-h indicates a poor prognosis in colorectal cancer, and is positively correlated with IRX5 mRNA expression. Onco Targets Ther. 2016;9:1437-1448. doi:10.2147/ OTT.S98268
16. Liu $\mathrm{T}$, Zhang $\mathrm{X}$, Gao $\mathrm{S}$, et al. Exosomal long noncoding RNA CRNDE-h as a novel serum-based biomarker for diagnosis and prognosis of colorectal cancer. Oncotarget. 2016;7(51):85551-85563. doi:10.18632/oncotarget.13465

17. Han P, Li JW, Zhang BM, et al. The lncRNA CRNDE promotes colorectal cancer cell proliferation and chemoresistance via miR-181a-5p-mediated regulation of Wnt/beta-catenin signaling. Mol Cancer. 2017;16(1):9. doi:10.1186/s12943-017-0583-1

18. Ding J, Li J, Wang H, et al. Long noncoding RNA CRNDE promotes colorectal cancer cell proliferation via epigenetically silencing DUSP5/CDKN1A expression. Cell Death Dis. 2017;8(8):e2997. doi:10.1038/cddis. 2017.518

19. Li Y, Huang W, Liu J, et al. The predictive value of serum IncRNA CRNDE for efficacy of first-line FOLFOX chemotherapy in patients with metastatic colorectal cancer and their prognosis. Dept Oncol. 2018;16(07):897-900+916.

20. Du DX, Lian DB, Amin BH, Yan W. Long non-coding RNA CRNDE is a novel tumor promoter by modulating PI3K/AKT signal pathways in human gastric cancer. Eur Rev Med Pharmacol Sci. 2017;21 (23):5392-5398. doi:10.26355/eurrev_201712_13925

21. Wang G, Pan J, Zhang L, Li Q, Tao Z, Lin Y. Long non-coding RNA CRNDE sponges miR-384 to promote proliferation and metastasis of pancreatic cancer cells through upregulating IRS1. Cell Prolif. 2017;50(6). doi:10.1111/cpr.12368

22. Xia XL, Xue D, Xiang TH, et al. Overexpression of long non-coding RNA CRNDE facilitates epithelial-mesenchymal transition and correlates with poor prognosis in intrahepatic cholangiocarcinoma. Oncol Lett. 2018;15(4):4105-4112. doi:10.3892/ol.2018.7815

23. Tierney JF, Stewart LA, Ghersi D, Burdett S, Sydes MR. Practical methods for incorporating summary time-to-event data into meta-analysis. Trials. 2007;8:16. doi:10.1186/1745-6215-8-16

24. Parmar MK, Torri V, Stewart L. Extracting summary statistics to perform meta-analyses of the published literature for survival endpoints. Stat Med. 1998;17(24):2815-2834.

25. Lin C, Yang L. Long noncoding RNA in cancer: wiring signaling circuitry. Trends Cell Biol. 2018;28(4):287-301. doi:10.1016/j.tcb.2017.11.008

26. Zhang M, Gao C, Yang Y, et al. Long noncoding RNA CRNDE/ PRC2 participated in the radiotherapy resistance of human lung adenocarcinoma through targeting p21 expression. Oncol Res. 2018;26(8):1245-1255. doi:10.3727/096504017X149445585873668

27. Qu L, Ding J, Chen C, et al. Exosome-transmitted lncARSR promotes sunitinib resistance in renal cancer by acting as a competing endogenous RNA. Cancer Cell. 2016;29(5):653-668. doi:10.1016/j.ccell.2016.03.004

28. Xie H, Ma B, Gao Q, et al. Long non-coding RNA CRNDE in cancer prognosis: review and meta-analysis. Clin Chim Acta. 2018;485:262-271. doi:10.1016/j.cca.2018.07.003

29. Liang C, Zhang B, Ge H, Xu Y, Li G, Wu J. Long non-coding RNA CRNDE as a potential prognostic biomarker in solid tumors: a meta-analysis. Clin Chim Acta. 2018;481:99-107. doi:10.1016/j. cca.2018.02.039

30. Yu B, Ye X, Du Q, et al. The long non-coding RNA CRNDE promotes colorectal carcinoma progression by competitively binding miR-217 with TCF7L2 and enhancing the wnt/beta-catenin signaling pathway. Cell Physiol Biochem. 2017;41(6):2489-2502. doi:10.1159/000475941

31. Gao H, Song X, Kang T, et al. Long noncoding RNA CRNDE functions as a competing endogenous RNA to promote metastasis and oxaliplatin resistance by sponging miR-136 in colorectal cancer. Oncol Targets Ther. 2017;10:205-216. doi:10.2147/OTT.S116178

32. Chen Z, Yu C, Zhan L, Pan Y, Chen L, Sun C. LncRNA CRNDE promotes hepatic carcinoma cell proliferation, migration and invasion by suppressing miR-384. Am J Cancer Res. 2016;6(10):2299-2309.

33. Wang H, Ke J, Guo Q, Barnabo Nampoukime K-P, Yang P, Ma K. Long non-coding RNA CRNDE promotes the proliferation, migration and invasion of hepatocellular carcinoma cells through miR-217/ MAPK1 axis. J Cell Mol Med. 2018;22:5862-5876. doi:10.1111/ jcmm.2018.22.issue-12 


\section{Supplementary materials}

A

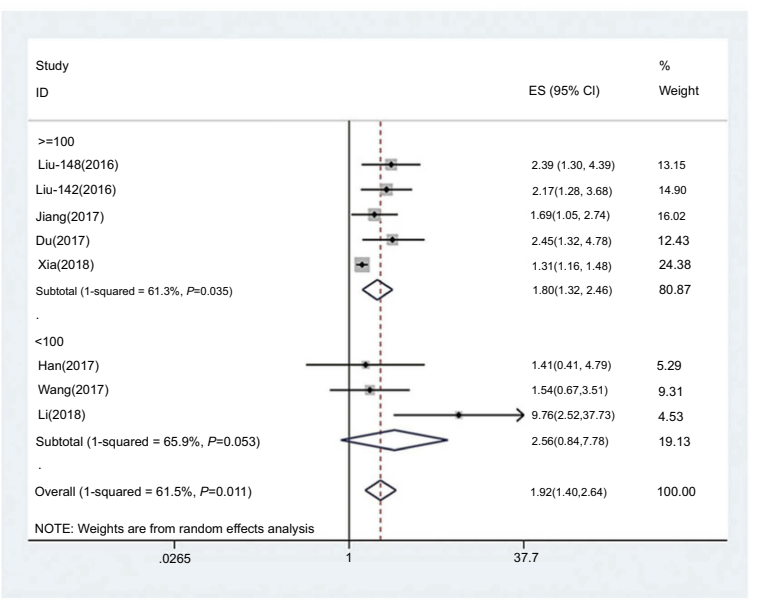

C

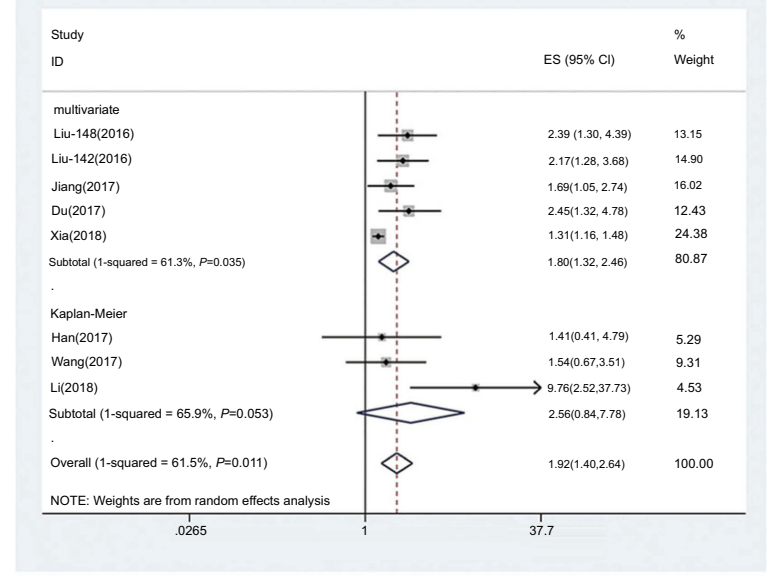

B

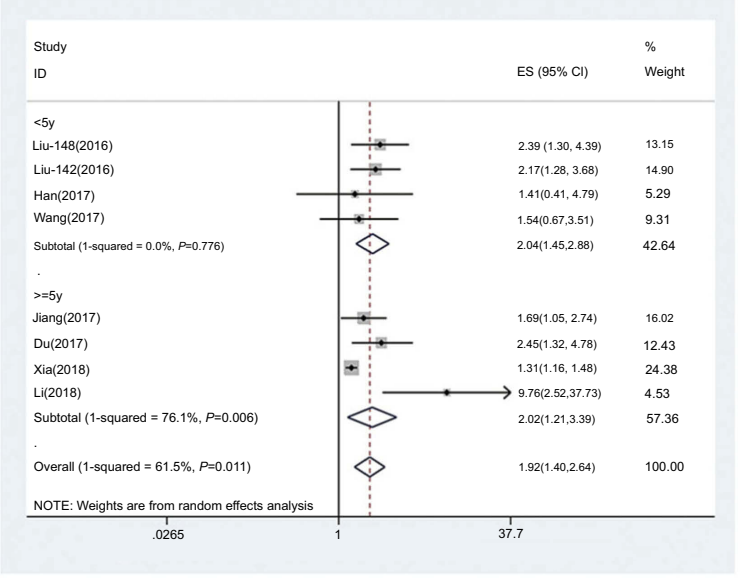

Figure SI Meta-analysis of the pooled HRs of OS in different types of cancer with high CRNDE expression. (A) Subgroup analysis of HRs of OS according to the factor of sample size. (B) Subgroup analysis of HRs of OS according to the factor of follow-up time. (C) Subgroup analysis of HRs of OS according to the factor of analysis type. Abbreviation: ES, effect size.

A

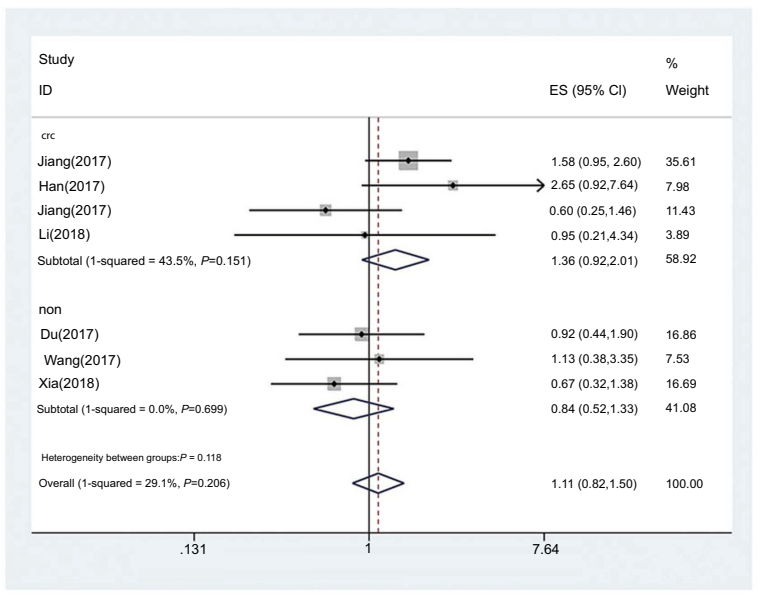

B

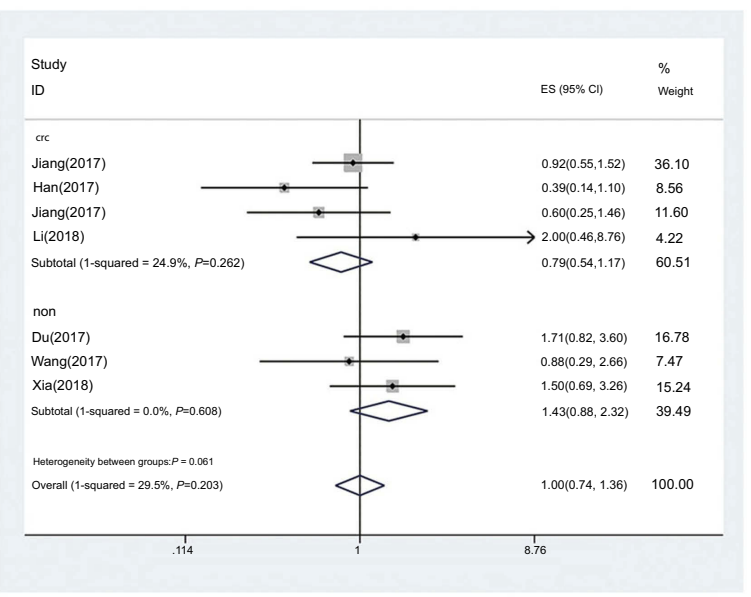

Figure S2 Forest plot of the correlation between CRNDE expression and clinicopathological characteristics. (A) Age (venerable age vs no). (B) Sex (female vs male). Abbreviations: CRC, colorectal cancer; non, non-colorectal cancer; ES, effect size. 


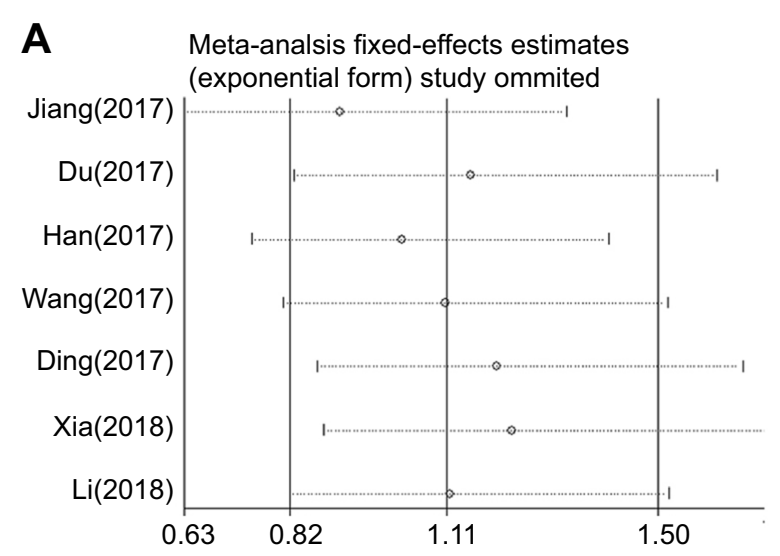

C Meta-analsis fixed-effects estimates

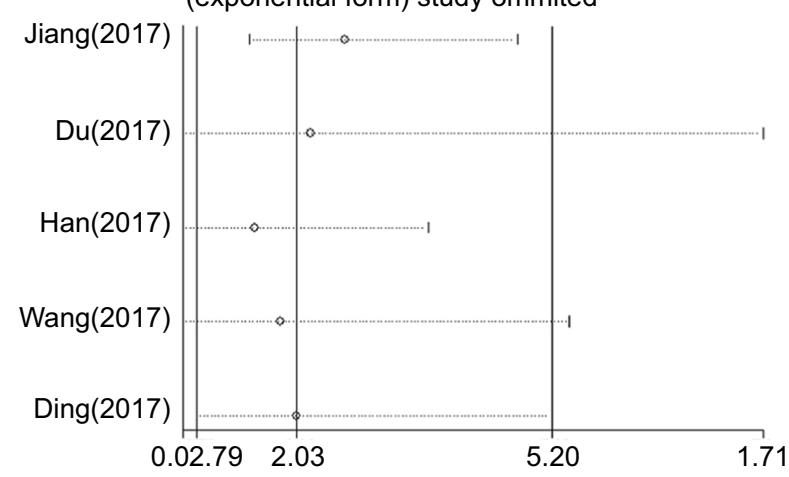

B Meta-analsis fixed-effects estimates (exponential form) study ommited

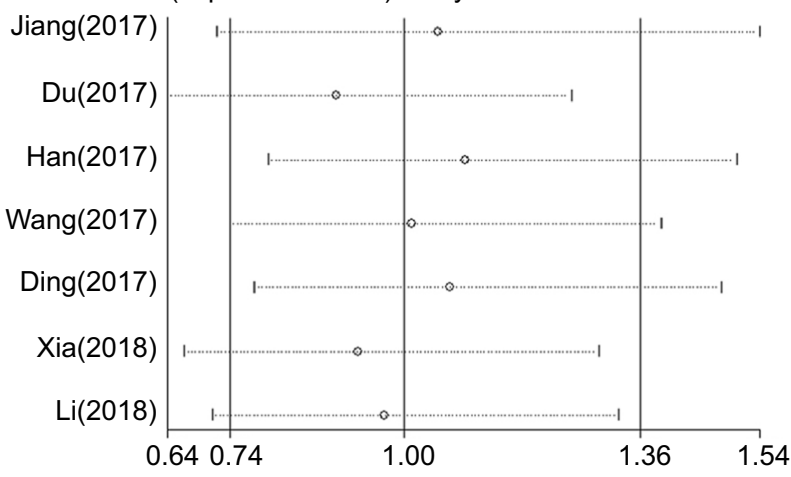

D Meta-analsis fixed-effects estimates

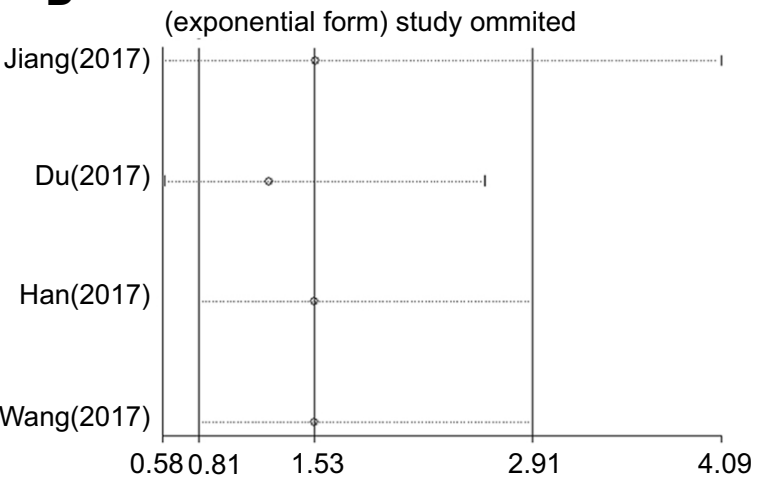



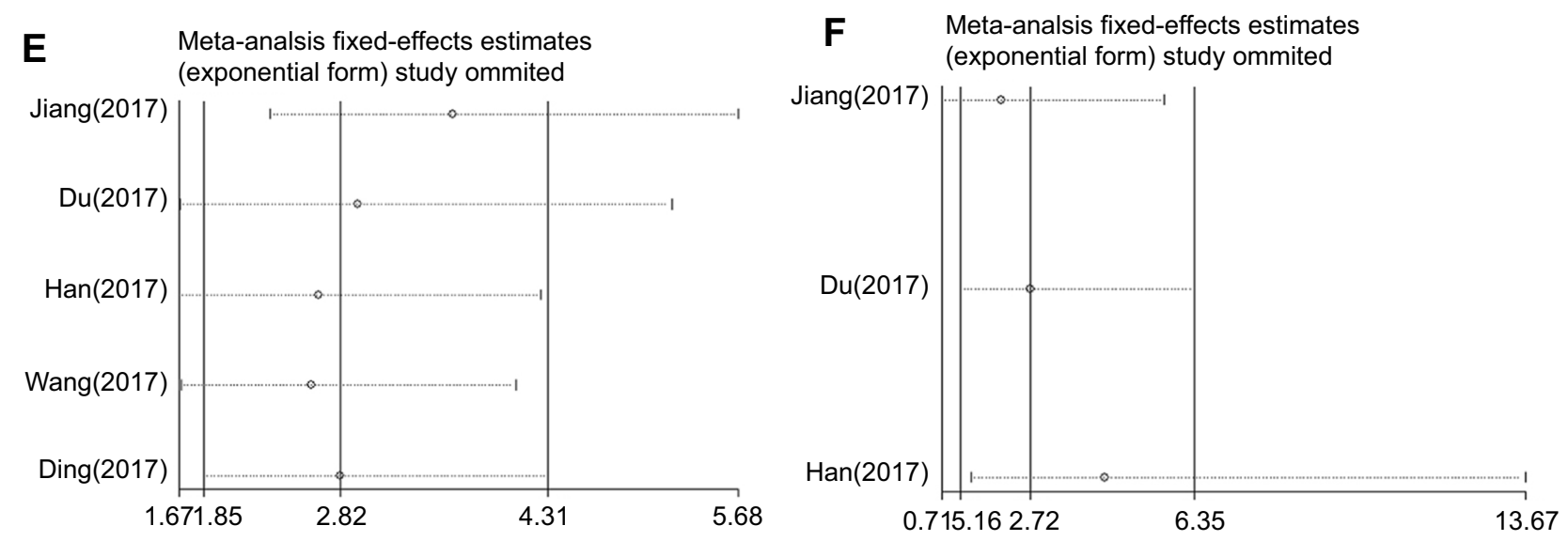

\section{G}

Meta-analsis fixed-effects estimates (exponential form) study ommited
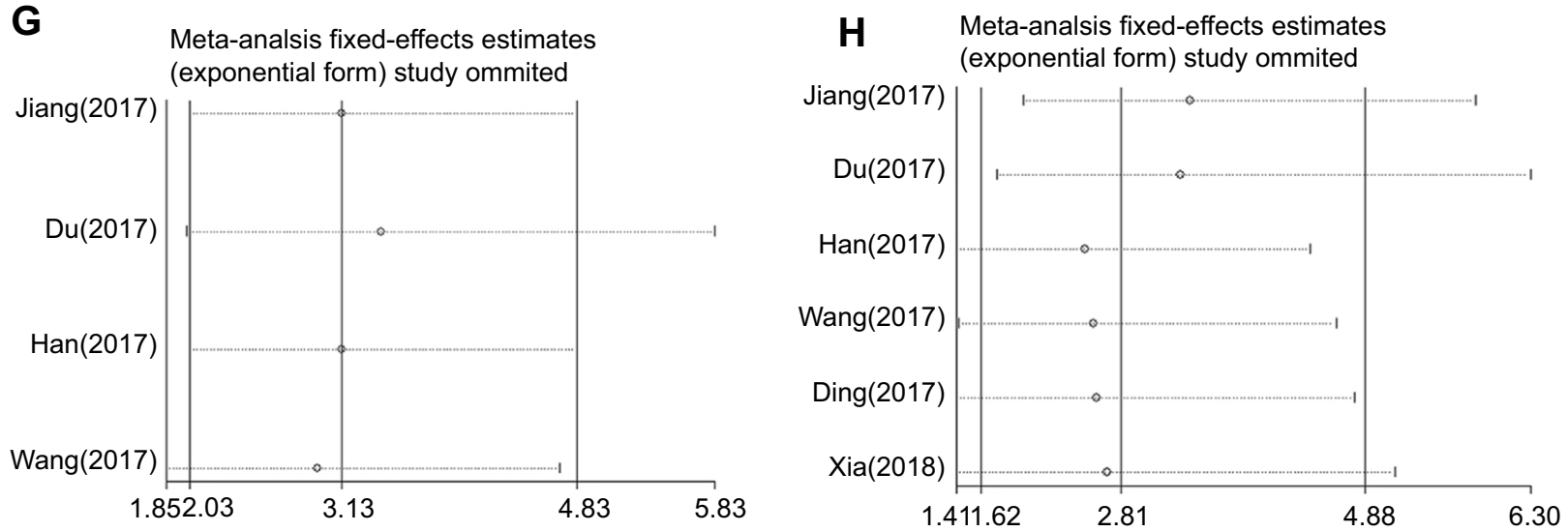

Figure S3 Sensitivity of CRNDE expression for the ORs of clinicopathological characteristics. (A) Age. (B) Sex. (C) Differentiation. (D) Depth of invasion. (E) Lymph node metastasis. (F) Distant metastasis. (G) TNM stage. (H) Tumor size.

Cancer Management and Research

\section{Dovepress}

\section{Publish your work in this journal}

Cancer Management and Research is an international, peer-reviewed open access journal focusing on cancer research and the optimal use of preventative and integrated treatment interventions to achieve improved outcomes, enhanced survival and quality of life for the cancer patient.
The manuscript management system is completely online and includes a very quick and fair peer-review system, which is all easy to use. Visit http://www.dovepress.com/testimonials.php to read real quotes from published authors. 